\title{
Line profile variability in the spectra of Oef stars
}

\section{I. $B D+60^{\circ} 2522^{\star}$}

\author{
G. Rauw* ${ }^{\star}$, M. De Becker, and J.-M. Vreux \\ Institut d'Astrophysique et de Géophysique, Université de Liège, Allée du 6 Août, Bât. B5c, 4000 Liège (Sart Tilman), Belgium \\ Received 5 November 2002 / Accepted 14 November 2002

\begin{abstract}
We report the analysis of a long term observing campaign designed to monitor the spectroscopic variability of the Oef star BD $+60^{\circ} 2522$. We find that the double-peaked He II $\lambda 4686$ emission line undergoes strong profile variability on time scales of 2-3 day. However, the time scale as well as the pattern of these variations turn out to be epoch dependent and the phenomenon is thus most likely not ruled by a single stable clock. On the other hand, the absorption lines in the spectrum of $\mathrm{BD}+60^{\circ} 2522$ display line profile variability on time scales of a few hours that might be related to non-radial pulsations. We tentatively propose that the beating of several non-radial pulsation modes triggers transient large-scale density perturbations in a confined stellar wind that produce the $2-3$ day time scale variability.
\end{abstract}

Key words. line: profiles - stars: early-type - stars: mass-loss - stars: individual: BD $+60^{\circ} 2522$ - stars: variables: general

\section{Introduction}

Almost thirty years ago, Walborn (1973) and Conti \& Leep (1974) called attention to a group of Of stars where the He II $\lambda 4686$ emission line is found to display a double-peaked profile. These objects were classified as Onfp stars by Walborn (1973), whereas Conti \& Leep (1974) favored an Oef naming (we shall adopt the latter terminology throughout this paper). In both papers, it was pointed out that the stars in this category are rapid rotators (as derived from their broad absorption lines) and that the occurrence of the Oef phenomenon may be related to rotation. More specifically, Conti \& Leep (1974) suggested that the peculiar morphology of the He II $\lambda 4686$ profiles results from a combination of a roughly central emission and a slightly blueshifted absorption in stars having stellar winds with appreciable rotational rates (see also Petrenz \& Puls 1996).

Oef stars usually do not display emission in the Balmer lines, but they have broad absorption lines indicative of large projected rotational velocities. All known Oef stars are of early spectral type. Conti \& Leep (1974) listed a total of six stars belonging to this category; all of them, except $\zeta$ Pup, being located in the northern hemisphere. The brightest and hence best studied representatives of this group are $\zeta$ Pup (O4 ef) and $\lambda$ Cep (O6 ef). The remaining four objects (HD 14434, HD 14442, HD 192281 and $\mathrm{BD}+60^{\circ}$ 2522) have received little attention over the last three decades.

Send offprint requests to: $\mathrm{G}$. Rauw,

e-mail: rauw@astro.ulg.ac.be

* Based on observations collected at the Observatoire de Haute Provence, France.

$\star \star$ Research Associate FNRS (Belgium).
Considerable progress in our understanding of the stellar winds of early-type stars has been achieved through extensive monitoring of their spectroscopic variability and the discovery that some of the cyclical variations could be related to a rotational modulation of the stellar wind (for a review, see e.g. Fullerton 1999). Since rotation is believed to shape the winds of Oef stars, these objects appear a priori as good candidates to search for a rotational wind modulation. In fact, part of the line profile variability observed in the spectra of $\zeta$ Pup and $\lambda$ Cep has been attributed to the effect of rotation (Moffat \& Michaud 1981; Howarth et al. 1995; Kaper et al. 1999). In order to investigate the spectroscopic variability of the other, poorly known, Oef stars, we have started an extensive observing campaign to collect spectra of these objects. In the present paper, we focus on the results of our seven year observing campaign of $\mathrm{BD}+60^{\circ} 2522$. The spectroscopic variability of HD 14434 , HD 14442 and HD 192281 will be discussed in a forthcoming paper (De Becker et al. 2003, in preparation).

$\mathrm{BD}+60^{\circ} 2522(V=8.7)$ is the ionizing star of NGC 7635, the so-called "Bubble Nebula" (see e.g. Christopoulou et al. 1995). NGC 7635 lies at the edge of a low-density clumpy molecular cloud and the nebula can be interpreted as a wind-blown bubble created by the interaction of the stellar wind of $\mathrm{BD}+60^{\circ} 2522$ with the ambient interstellar medium (Christopoulou et al. 1995; Chavarría-K. et al. 1987). While many investigations have focused on the nebula, little attention has been paid to the star itself.

The paper is organized as follows. Section 2 briefly summarizes our observing campaign and the data reduction. In Sect. 3 we present the results of our radial velocity and line profile 
variability analyses. The origin of the line profile variability is discussed in Sect. 4 and our conclusions are highlighted in Sect. 5.

\section{Observations and data reduction}

Spectroscopic observations of $\mathrm{BD}+60^{\circ} 2522$ were collected during several observing campaigns between August 1996 and September 2002 at the Observatoire de Haute-Provence (OHP).

Our observations were obtained with the Aurélie spectrograph fed by the $1.52 \mathrm{~m}$ telescope at OHP (Gillet et al. 1994). Until 1999, Aurélie was equipped with a Thomson TH7832 linear array with a pixel size of $13 \mu \mathrm{m}$. From 2000 on, the detector of the Aurélie instrument was replaced by a $2048 \times 1024$ CCD EEV 42-20\#3, with a pixel size of $13.5 \mu \mathrm{m}$ squared.

Table 1 provides an overview of the various instrumental configurations. All the data were reduced in the standard way using the MIDAS package developed at ESO. The spectra were normalized using properly chosen continuum windows.

Table 1 also yields some information about the sampling of the various observing campaigns. For each campaign, $\Delta T$ gives the total time elapsed between our first and our last observation, while $N$ is the total number of observations. $\overline{\Delta t}$ provides the average time interval between two consecutive exposures during the same night. In light of the Fourier analysis, Table 1 further lists the natural width of a peak in the power spectrum $\Delta v_{\text {nat }}=1 / \Delta T$, while $v_{\max }=1 /(2 \overline{\Delta t})$ provides a rough indication of the highest frequencies that could be sampled with our data set.

\section{Results}

\subsection{The spectrum of $B D+60^{\circ} 2522$}

The blue-violet spectrum of $\mathrm{BD}+60^{\circ} 2522$ is shown in Fig. 1 . Besides the typical absorption lines of $\mathrm{HI}_{\mathrm{I}} \mathrm{He}_{\mathrm{I}}$ and $\mathrm{He}$ II, this spectral domain contains the complex emission blend of $\mathrm{N}$ III $\lambda \lambda 4634-41$ and $C_{\text {III }} \lambda \lambda 4647-50$ as well as the double-peaked He II $\lambda 4686$ emission. The latter emission lines are found on top of a broad emission extending from about 4610 to $4720 \AA$, a feature that is quite common among Of stars (Underhill et al. 1989). We also note the faint Of emission lines at 4485 and $4505 \AA$ attributed to S Iv by Werner \& Rauch (2001). We caution that the Balmer absorption lines might be contaminated by nebular emission from the Bubble Nebula.

The ratio of the equivalent widths $(E W s)$ of the He I $\lambda 4471$ and He II $\lambda 4542 \operatorname{lines}, \log W^{\prime}=\log (E W(\mathrm{HeI} \lambda 4471) /$ $E W($ HeII $\lambda 4542))=-0.18 \pm 0.03$, as measured on our spectra (Table 2), points towards an O6.5 spectral type for $\mathrm{BD}+60^{\circ} 2522$ according to the classification scheme of Conti (1973). This result is in excellent agreement with previous classifications. Doroshenko (1972) inferred an O5 spectral type ${ }^{1}$, while Walborn (1973) and Conti \& Leep (1974) classified $\mathrm{BD}+60^{\circ} 2522$ as $\mathrm{O6.5}(\mathrm{n})(\mathrm{f}) \mathrm{p}$ and O6.5 IIIef respectively.

\footnotetext{
1 Although we note that the equivalent widths of the He s $\lambda 4471$ and He II $\lambda 4542$ lines quoted by Doroshenko (1972) yield an O5.5 spectral type according to the classification criterion introduced lateron by Conti (1973).
}

The giant luminosity class quoted by Conti \& Leep was first proposed by Conti \& Alschuler (1971). Since none of the conventional luminosity criteria (mainly based on the nature and strength of He II $\lambda$ 4686) can be applied to BD $+60^{\circ} 2522$ in a straightforward manner, we refrain from proposing a luminosity class ourselves.

\subsection{Radial velocities}

Doroshenko (1972) noted an apparently “abnormal energy distribution" and suggested that this could be the signature of a binary system with the secondary being of spectral type F5 Ib. Subsequent investigations revealed however that flat energy distributions are common among Of stars and can be attributed to the effect of atmospheric extension (see e.g. Kuan \& Kuhi 1976).

To investigate the multiplicity of $\mathrm{BD}+60^{\circ} 2522$, we have measured the radial velocities (RVs) of the two most prominent absorption lines in the 4455-4905 $\AA$ domain (i.e. He I $\lambda 4471$ and He II $\lambda 4542$ ) by fitting Gaussians. The results indicate a rather small range of variability. For the He I $\lambda 4471$ and He II $\lambda 4542$ lines we find standard deviations around the mean RV of respectively 9.4 and $7.1 \mathrm{~km} \mathrm{~s}^{-1}$.

We applied a Fourier analysis to the entire $R V$ data set of each of the two absorption lines. The most prominent peaks in the power spectra occur at frequencies below $\sim 0.03 \mathrm{~d}^{-1}$. The amplitudes of the sine waves fitted to the data at the "highestpeak" frequencies are 7.2 and $5.4 \mathrm{~km} \mathrm{~s}^{-1}$ respectively for $\mathrm{He}$ I $\lambda 4471$ and He II $\lambda 4542$. These amplitudes are of the same order as the typical errors on a single $R V$ point $\left(\sim 10 \mathrm{~km} \mathrm{~s}^{-1}\right.$ for the $33 \AA \mathrm{mm}^{-1}$ data) and we do not regard them to be significant. Moreover, both absorption lines undergo profile variations (see Sect. 3.3.2) that may account for the small amplitude $R V$ variations discussed here.

Since no significant short-term variability was found, we have averaged the $R V \mathrm{~s}$ from each observing campaign (see Table 2). Most of these values overlap within the errors with the mean of the entire data set (Fig. 2).

In summary, there is no consistent and significant periodicity in the RVs of the different absorption lines and although we cannot completely rule out a long period binary system seen under a very low inclination angle, it seems more likely that $\mathrm{BD}+60^{\circ} 2522$ is indeed a single star.

\subsection{Line profile variability}

To identify the features in the spectrum of $\mathrm{BD}+60^{\circ} 2522$ that display significant variability, we have computed the time variance spectrum (TVS, Fullerton et al. 1996) of our entire data set over the spectral range 4465 to $4800 \AA$. The largest amplitude of the TVS is found over the He II $\lambda 4686$ line. Significant variability occurs also in the $\mathrm{He}$ I $\lambda 4471$ and $\mathrm{He}$ II $\lambda 4542$ lines. On the other hand, the TVS of the entire data set yields some marginal variability in the $\mathrm{N}$ III $\lambda \lambda 4634-41$ and $\mathrm{C}$ III $\lambda \lambda 4647$ 50 blend, but this variability disappears when we consider the TVS of individual observing campaigns. The variability in the entire data set could either result from long term changes or 
Table 1. Overview of our observing campaigns. The third column indicates the detector used with the Aurélie spectrograph (see text). $N$ yields the number of spectra. $\Delta T, \overline{\Delta t}, \Delta v_{\text {nat }}$ and $v_{\text {max }}$ provide for each campaign respectively the total time elapsed between the first and the last observation, the average time interval between consecutive exposures during the same night, the natural width of a peak in the power spectrum and the highest frequency that can be sampled with our data set (see text). $(S / N)_{0}$ stands for the average signal to noise ratio evaluated over a line-free region of the spectrum.

\begin{tabular}{llccccccccc}
\hline \hline Run & Epoch & Detector & $\begin{array}{c}\text { Spectral range } \\
(\AA)\end{array}$ & $\begin{array}{c}\text { Recipr. disp. } \\
\left(\AA \mathrm{Amm}^{-1}\right)\end{array}$ & $N$ & $\begin{array}{c}\Delta T \\
\text { days }\end{array}$ & $\begin{array}{c}\overline{\Delta t} \\
\text { days }\end{array}$ & $\begin{array}{c}\Delta v_{\text {nat }} \\
\left(\mathrm{d}^{-1}\right)\end{array}$ & $\begin{array}{c}v_{\max } \\
\left(\mathrm{d}^{-1}\right)\end{array}$ & $(S / N)_{0}$ \\
\hline [1] & Aug. 1996 & TH7832 & $4100-4950$ & 33 & 13 & 3.124 & 0.051 & 0.320 & 9.8 & 320 \\
[2] & Jul. 1997 & TH7832 & $4100-4950$ & 33 & 18 & 6.109 & 0.044 & 0.164 & 11.4 & 430 \\
[3] & Sep. 1998 & TH7832 & $4455-4905$ & 16 & 6 & 9.008 & 0.058 & 0.111 & 8.6 & 230 \\
[4] & Jul. 1999 & TH7832 & $4100-4950$ & 33 & 14 & 6.029 & 0.040 & 0.166 & 12.5 & 215 \\
[5] & Aug. 1999 & TH7832 & $4100-4950$ & 33 & 12 & 13.008 & - & 0.077 & 1.0 & 210 \\
[6] & Sep. 2000 & CCD & $4455-4905$ & 16 & 18 & 10.998 & 0.024 & 0.091 & 20.8 & 470 \\
[7] & Sep. 2001 & CCD & $4455-4905$ & 16 & 14 & 7.053 & 0.026 & 0.142 & 19.2 & 320 \\
{$[8]$} & Sep. 2002 & CCD & $4455-4905$ & 16 & 9 & 5.006 & 0.028 & 0.200 & 17.6 & 380 \\
\hline
\end{tabular}

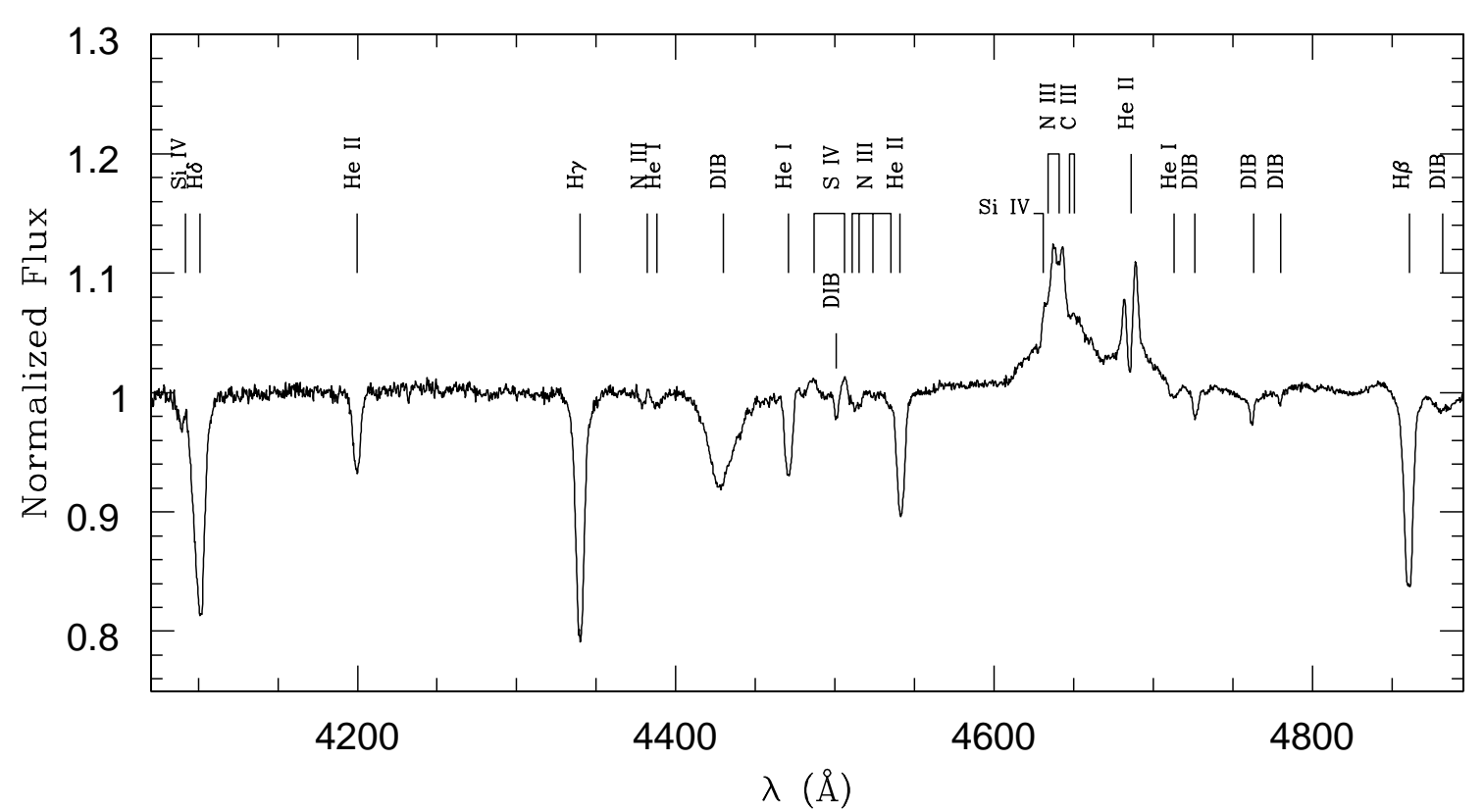

Fig. 1. Mean spectrum of BD +60 2522 as observed in August 1996.

from small normalization discrepancies between the various data sets. Indeed, some variability is also detected over the narrow diffuse interstellar bands (DIBs) at 4502, 4726, 4763 and $4780 \AA$. The apparent variability in these features most probably results from small normalization discrepancies between different observing campaigns. During a single observing campaign, these normalization errors are always limited to $T V S^{1 / 2} \leq 0.004$.

\subsubsection{Profile variability of the $\mathrm{He}$ II $\lambda 4686$ line}

A simple inspection of the data during the observing runs suggested that the He II $\lambda 4686$ line varies on time scales of a few days (see Fig.4). However, when we compare the variations as observed during the various campaigns, we find significant epoch-dependent differences (see Fig. 5).

The overall level of profile variability changes with time. Our TVS variability estimator reached its maximum in
July 1999 and September 2000, whereas it was lowest in August 1996. Also the wavelength range over which the $T V S^{1 / 2}$ exceeds the $99 \%$ significance level varied from one observing campaign to the other and reached its largest extent in September 2000. But most of all, it is the morphology of the $T V S^{1 / 2}$ that varies. During observing campaigns [1], [2], [7] and [8], the variability reaches its maximum in the core of the absorption component, whereas the TVS is larger over the emission components during campaigns [5] and [6].

If the line profile variability of the $\mathrm{He}$ II $\lambda 4686$ line were periodic, some of the differences discussed hereabove might actually result from the sampling of the cycle during the various observing campaigns. We have applied the 2-D Fourier techniques described by Rauw et al. (2001) to the time series of each campaign. The results are indicated in Table 3 below. Most of the time the periodograms display one dominant peak together with its one-day aliases. However, in August 1999 (campaign [5]), we find no clearly dominant peak. 
Table 2. Properties of the $\mathrm{He}$ I $\lambda 4471$ and $\mathrm{He}$ II $\lambda 4542$ lines in the spectrum of $\mathrm{BD}+60^{\circ} 2522$. The second column yields the mean observation date in HJD-2450000. Typical standard deviations on the mean equivalent widths are of order $0.03 \AA$.

\begin{tabular}{cccccc}
\hline \hline Run & $\bar{T}$ & \multicolumn{2}{c}{$\mathrm{He}_{\mathrm{I}} \lambda 4471$} & \multicolumn{2}{c}{ He II $\lambda 4542$} \\
& & $\begin{array}{c}R V \\
\left(\mathrm{~km} \mathrm{~s}^{-1}\right)\end{array}$ & $\begin{array}{c}E W \\
(\AA)\end{array}$ & $\begin{array}{c}R V \\
\left(\mathrm{~km} \mathrm{~s}^{-1}\right)\end{array}$ & $\begin{array}{c}E W \\
(\AA)\end{array}$ \\
\hline$[1]$ & 312.1 & $-20.9 \pm 8.7$ & 0.39 & $1.1 \pm 6.3$ & 0.65 \\
{$[2]$} & 641.5 & $-14.3 \pm 4.9$ & 0.45 & $6.4 \pm 5.3$ & 0.61 \\
{$[3]$} & 1071.1 & $-20.0 \pm 5.3$ & 0.42 & $-2.7 \pm 8.6$ & 0.67 \\
{$[4]$} & 1376.5 & $-22.6 \pm 10.7$ & 0.46 & $-3.0 \pm 9.6$ & 0.66 \\
{$[5]$} & 1403.7 & $-28.3 \pm 8.9$ & 0.45 & $-1.1 \pm 5.7$ & 0.67 \\
{$[6]$} & 1815.7 & $-28.9 \pm 7.7$ & 0.46 & $-5.1 \pm 3.8$ & 0.70 \\
{$[7]$} & 2167.0 & $-26.1 \pm 8.0$ & 0.46 & $-3.9 \pm 4.2$ & 0.72 \\
{$[8]$} & 2522.4 & $-28.8 \pm 8.8$ & 0.46 & $-8.6 \pm 2.8$ & 0.70 \\
\hline
\end{tabular}
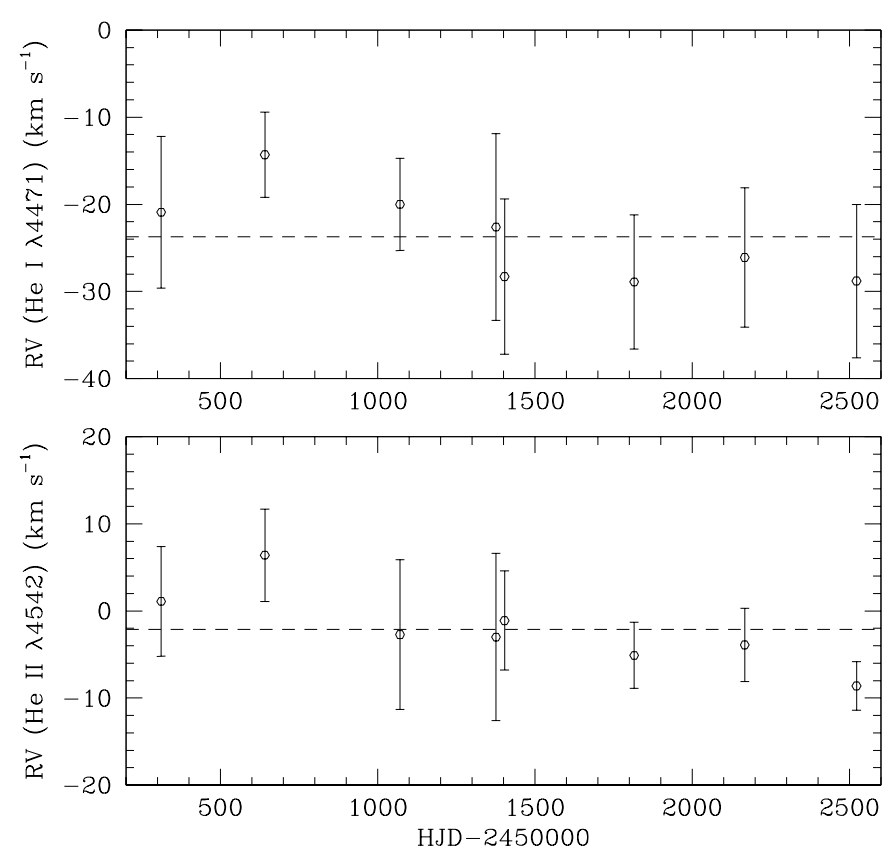

Fig. 2. Epoch-dependent mean $R V \mathrm{~s}$ of the He i $\lambda 4471$ and He II $\lambda 4542$ lines in the spectrum of $\mathrm{BD}+60^{\circ} 2522$. The dashed lines yield the average $R V$ over the entire data set. The error bars correspond to the $1-\sigma R V$ dispersion during a specific run.

Most intriguing is the fact that the position of the dominant peak changes from somewhere around $0.34 \mathrm{~d}^{-1}$ (or $0.66 \mathrm{~d}^{-1} \mathrm{de}-$ pending on which alias is the right one, if any) to somewhere between 0.40 and $0.45 \mathrm{~d}^{-1}$. To illustrate this situation, Fig. 6 displays the 2-D power spectra of three different data sets corresponding to a dominant peak at $0.34 \mathrm{~d}^{-1}$ (July 1997), no dominant peak (August 1999) and a dominant peak found in both emission components around $0.40 \mathrm{~d}^{-1}$ (September 2000).

Since each observing campaign contains only a limited number of data, one may wonder about the robustness of the Fourier analyses. Therefore, we have performed some trials to evaluate the impact of specific spectra on the aspect of the TVS

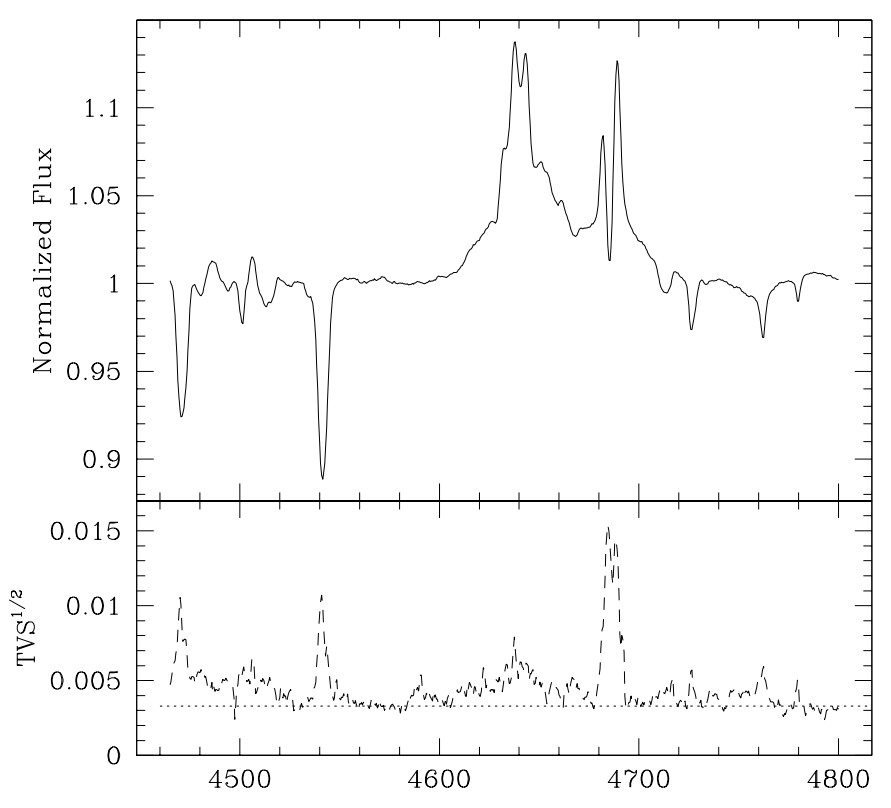

Fig. 3. Top panel: mean spectrum of $\mathrm{BD}+60^{\circ} 2522$ as observed with the Aurélie spectrograph at OHP from 1996 to 2002. Bottom panel: square root of the time variance spectrum. The dotted horizontal line corresponds to the threshold for variations significant at the $99 \%$ level for a total of 104 spectra and an average $S / N$ ratio of 350 (see Fullerton et al. 1996).

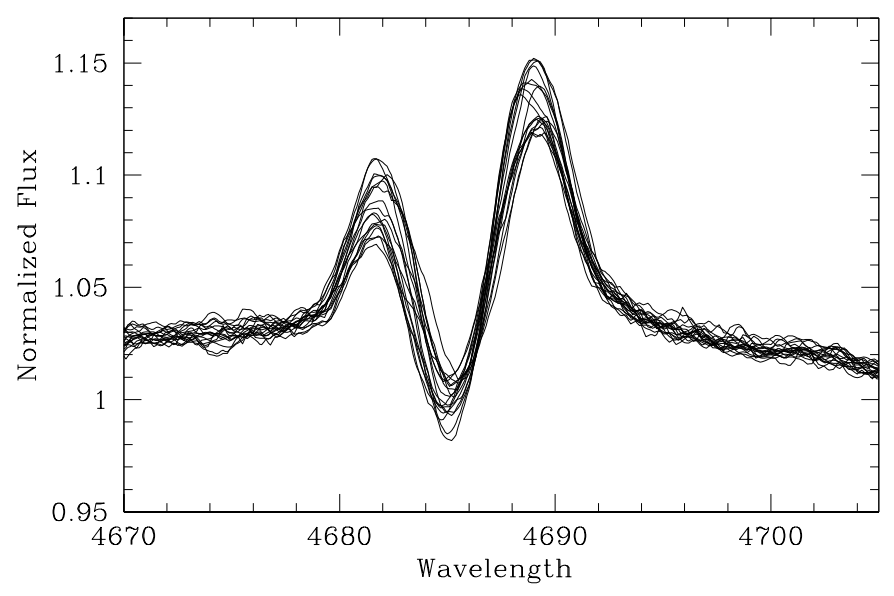

Fig. 4. Variations of the He II $\lambda 4686$ profile as observed in September 2000. During this campaign, the variations mainly resulted from changing relative intensities of the violet and red emission component.

and the location of the highest peaks in the periodograms. To do this, we have removed the different data points one at the time from the time series and repeated the analysis of the remaining data set. These tests showed that the periodograms are usually very stable except for campaigns [3], [5], [7] and [8] that turned out to be quite sensitive to the presence or absence of individual spectra. For campaigns [3] (September 1998) and [8] (September 2002) this is indeed not unexpected given the very limited number of spectra obtained during these runs. For campaign [5], we cannot blame the number of spectra or the sampling. In this latter data set, the periodogram does not reveal a clearly dominating peak and the poor stability of the 
Table 3. Properties of the 2-D periodogram of the time series consisting of the He II $\lambda 4686$ line profile as observed during various observing runs. The second column yields the maximum value of $T V S^{1 / 2}$ and the wavelength where this maximum occurs. The third column indicates the wavelength range over which $T V S^{1 / 2}$ exceeds the $99 \%$ significance level. The fourth and fifth columns yield the highest and second highest peaks in the periodogram over the $[0.0,1.0] \mathrm{d}^{-1}$ frequency range. The last column provides the mean equivalent width of the line as integrated between 4675 and $4700 \AA$. The maximum $1-\sigma$ dispersion of this $E W$ during a specific run amounts to $\sim 0.1 \AA$.

\begin{tabular}{cccccc}
\hline \hline Run & $T V S_{\max }^{1 / 2}$ & $\begin{array}{c}\lambda-\text { range } \\
(\AA)\end{array}$ & $\begin{array}{c}v_{1} \\
\mathrm{~d}^{-1}\end{array}$ & $\begin{array}{c}v_{2} \\
\mathrm{~d}^{-1}\end{array}$ & $\begin{array}{c}\overline{E W} \\
(\AA)\end{array}$ \\
\hline$[1]$ & 0.009 at 4685.1 & $4683-4691$ & 0.66 & 0.33 & -1.26 \\
{$[2]$} & 0.014 at 4686.4 & $4681-4693$ & 0.34 & 0.66 & -1.48 \\
{$[4]$} & 0.017 at 4684.3 & $4681-4690$ & 0.45 & 0.55 & -1.38 \\
{$[5]$} & 0.013 at 4684.1 & $4683-4690$ & $0.35^{a}$ & $0.65^{a}$ & -1.46 \\
{$[6]$} & 0.016 at 4683.2 & $4679-4692$ & 0.40 & 0.60 & -1.42 \\
{$[7]$} & 0.015 at 4684.6 & $4682-4690$ & $0.43^{b}$ & $0.57^{b}$ & -1.24 \\
{$[8]$} & 0.013 at 4686.9 & $4682-4692$ & $0.42^{a, c}$ & $0.85^{a, c}$ & -1.38 \\
\hline
\end{tabular}

${ }^{a}$ There are a number of other "peaks" in the periodogram that have only slightly lower amplitudes than $v_{1}$ and $v_{2}$.

${ }^{b}$ These frequencies correspond to the highest peaks over the wavelength interval 4682-4685. Over the entire range given in Col. 3, the highest peaks are found at 0.13 and $0.86 \mathrm{~d}^{-1}$.

${ }^{c}$ The periodogram for this run is very sensitive to the inclusion of individual spectra in the time series and the highest peak frequencies are very unstable.

periodogram is yet another indication that the variability was most probably not periodic in August 1999. Finally, the periodogram of the September 2001 campaign ([7]) turned out to be quite sensitive to the spectra obtained during the night of September 11-12 (HJD 2452165.431 and 2452165.455). If we omit these data from our analysis, we recover peaks at 0.34 and $0.66 \mathrm{~d}^{-1}$, pretty close to those found for campaigns [1] and [2]. However, there is no reason a priori to discard these data.

In the case of the He II $\lambda 4686$ line profile in the spectrum of $\mathrm{BD}+60^{\circ} 2522$, it seems therefore more appropriate to talk about time scales rather than periodicities. Indeed, a search for a stable coherent periodicity over the entire data set turned out to be illusory: the periodogram of the combined data set is dominated by very low frequencies and their daily aliases. The same conclusion holds for the $\mathrm{He}$ I $\lambda 4471$ and He II $\lambda 4542$ lines (see below). Also interesting to note is the fact that observations separated by only one month (July and August 1999) produce different power spectra. Finally, we note that the periodograms usually do not reveal strong peaks at frequencies higher than $1.0 \mathrm{~d}^{-1}$, except for some daily aliases of the peaks from the $[0.0,1.0] \mathrm{d}^{-1}$ interval.

Another illustration of the epoch dependence of the He II $\lambda 4686$ profile variations can be obtained by fitting an expression

$F(\lambda, t)=c_{0}(\lambda)+A(\lambda) \sin \left(2 \pi v_{1}\left[t-t_{0}\right]+\phi_{0}(\lambda)\right)$ to the time series of an individual campaign at every wavelength step. Here, $v_{1}$ corresponds to the dominant peak found in the Fourier analysis of the specific data set, while $c_{0}(\lambda), A(\lambda)$ and $\phi_{0}(\lambda)$ are fit parameters that represent the mean profile, the amplitude of variability and the phase constant at wavelength $\lambda$ respectively (see also Rauw et al. 2001). These parameters are illustrated in Fig. 7 for observing campaigns [2] and [6]. We remind the reader that the phase constant $\phi_{0}$ has physical meaning only if the period is clearly detected at the specific wavelength.

This reconstruction technique reveals that the profile modulation in July 1997 is mainly concentrated in the central absorption component (4683-4689 $\AA$ ). In addition the phase of the modulation $\phi_{0}$ remains roughly constant over this wavelength interval. Therefore, the variations of the profile in July 1997 can be described as a sinusoidal modulation of the depth of the absorption component with a "period" of 2.94 days. Such a variation might result for instance from a periodic modulation of the optical depth along the line of sight towards the stellar core.

On the contrary, the September 2000 data reveal a completely different picture. The amplitude peaks in the emission components of the line and $\phi_{0}$ varies strongly across the peaks of the amplitude. In summary, the variations in September 2000 can be described as a modulation of the strengths of the two emission peaks with a "period" of 2.50 days; the red peak reaching its maximum intensity when the violet peak is minimum and vice versa.

\subsubsection{Profile variability of the absorption lines}

We have applied the Fourier techniques to the profiles of the He I $\lambda 4471$ and He II $\lambda 4542$ absorption lines. It turns out that the level of variability during a specific observing run is usually rather low $\left(T V S^{1 / 2} \leq 0.007\right.$ on average). However, visual inspection of the spectra indicates profile variability in the line cores for those nights where we have obtained spectra separated by several hours (this is essentially the case for the August 1996 campaign, see Fig. 8, and for one night of the September 2002 run).

While the Fourier analysis of the entire data set fails to reveal a stable period, we find nevertheless that the Fourier periodograms of individual campaigns suggest that the line cores are variable on rather short time scales of the order of hours. Unfortunately, our data set was designed to search for variability on time scales of days rather than hours and hence does not allow us to study these short term variations in detail. From the analysis of the best suited data set (August 1996), we tentatively propose a time scale of about $4-5$ hours. A more intensive monitoring of the star with a good time resolution and a $\operatorname{good} S / N$ ratio is required to eventually establish the period(s) or time scale(s) of the phenomenon. Figure 8 suggests that the variations of the absorption line profiles may be due to nonradial pulsations (NRPs). This would not be surprising since NRPs have already been reported for the two brightest members of the Oef class (see Sect. 4 below). It seems very likely 

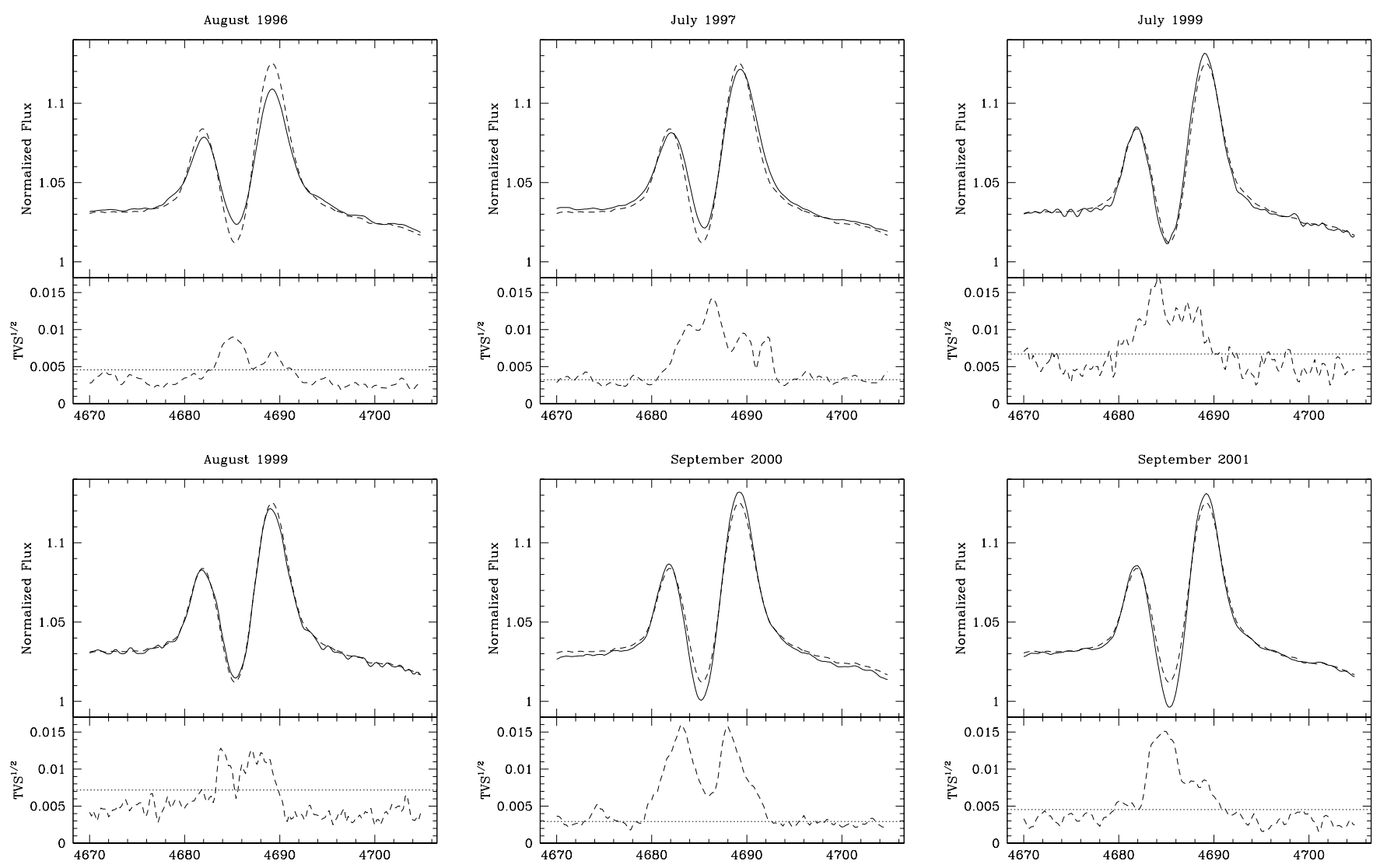

Fig. 5. The top three panels correspond to the variability of the He II $\lambda 4686$ line profile during campaigns [1], [2] and [4] from left to right, while the lower three panels correspond to campaigns [5], [6] and [7] (also from left to right). Each panel is divided into two subpanels. The upper subpanels yield the mean profiles as observed during a specific observing campaign (solid line) compared to the mean profile as evaluated from the entire data set (dashed line). The bottom subpanels yield the square root of the TVS while the dotted lines correspond to the thresholds for variations significant at the $99 \%$ level.

that the low amplitude $R V$ changes discussed in Sect. 3.2 are in fact a result of the absorption line profile variability.

\section{Discussion}

\subsection{Comparison with $\zeta$ Pup and $\lambda$ Cep}

At this stage it is worth comparing the variability of $\mathrm{BD}+60^{\circ} 2522$ with that of the two most prominent members of the Oef class: $\zeta$ Pup and $\lambda$ Cep.

Optical and UV observations of $\zeta$ Pup revealed appreciable variability on different time scales. Moffat \& Michaud (1981) and Howarth et al. (1995) detected a modulation of the stellar wind of $\zeta$ Pup with a period of 5.0-5.2 days thought to be the stellar rotation period. Moffat \& Michaud (1981) accordingly suggested that the inner regions of the stellar wind are forced into corotation by a magnetic field and $\zeta$ Pup could thus be a decentered oblique magnetic rotator.

In addition to the rotational modulation, the spectrum of $\zeta$ Pup displays also variations on two shorter time scales. The discrete absorption components (DACs) in the UV line profiles appear with a recurrence time of about $19 \mathrm{hrs}$ (Howarth et al. 1995). A perhaps related time scale of $16.7 \mathrm{hrs}$ was reported by Berghöfer et al. (1996) from an analysis of the variations of the $\mathrm{H} \alpha$ line and the ROSAT-PSPC count rate in the $0.9-2.0 \mathrm{keV}$ energy range. On the other hand, photospheric absorption lines exhibit moving bumps on time scales of $\sim 8.5 \mathrm{hrs}$. These features are usually interpreted as due to non-radial pulsations (e.g. Baade 1988; Reid \& Howarth 1996).

Finally, the existence of small scale clumpy structures in the wind of $\zeta$ Pup was suggested by Eversberg et al. (1998). These authors obtained a set of high quality high resolution spectra of the He II $\lambda 4686$ emission line that show stochastic variable substructures that they interpret as a manifestation of turbulent clumps propagating outward with the wind.

Henrichs (1991) reported covariability between the equivalent width of the He ir $\lambda 4686$ line and the blue steep edge of the $\mathrm{C}$ IV resonance lines in the spectrum of $\lambda \mathrm{Cep}$. He found a time scale of about two days for the variations of these lines, whereas the photospheric $\mathrm{He}$ I $\lambda 4713$ line displayed variations, likely due to non-radial pulsations, with a period of about 6.5 hrs. Variations in the $\mathrm{H} \alpha$ line on time scales of 4.8 days and 1.2 days were found by Kaper et al. (1997), whereas de Jong et al. (1999) confirmed the existence of NRPs with periods of 6.6 and $12.3 \mathrm{hrs}$. The latter authors suggested that beating among multimode NRPs could lead to cyclical surface amplitude enhancements that may eventually generate wind perturbations. 

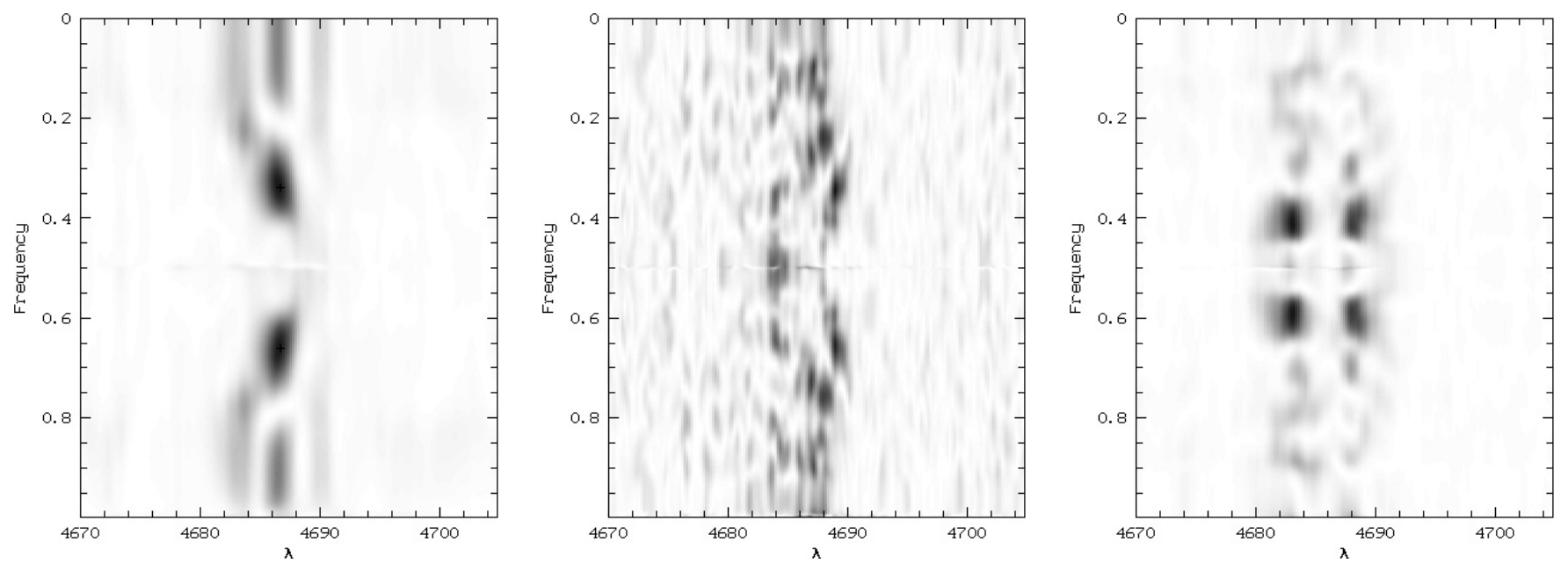

Fig. 6. Grey-scale images of the two-dimensional power spectra of the time series of the He II $\lambda 4686$ line in the frequency range [0.0, 1.0] $\mathrm{d}^{-1}$. The power spectra were computed using the Fourier technique described by Rauw et al. (2001). From left to right, the three panels correspond respectively to the data sets from July 1997 (campaign [2]), August 1999 (campaign [5]) and September 2000 (campaign [6]).
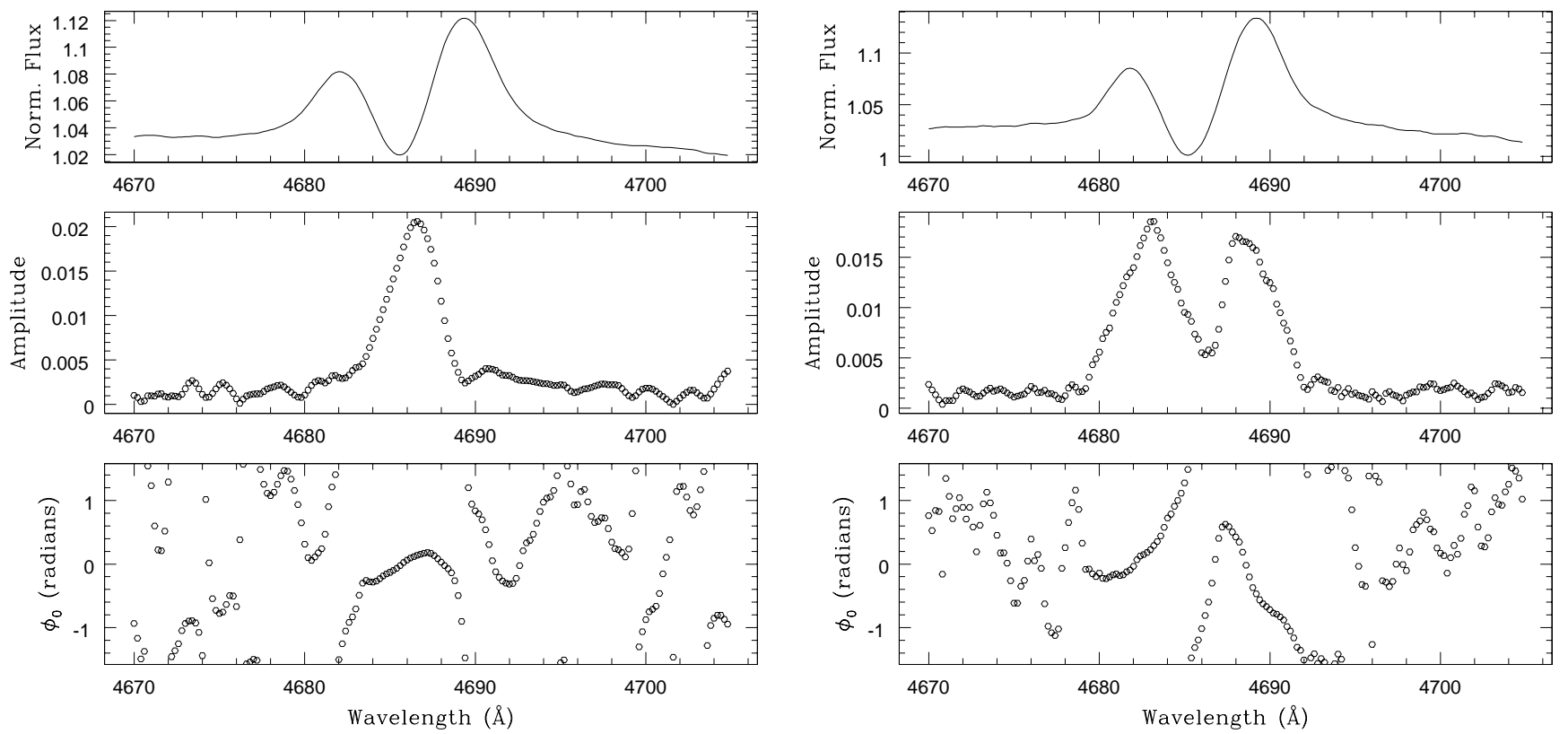

Fig. 7. Left: variability of the He II $\lambda 4686$ line in July 1997 (campaign [2]) as a function of phase assuming a "period" of 2.94 days $\left(v_{1}=\right.$ $0.34 \mathrm{~d}^{-1}$ ). The three panels display the mean line profile, as well as the amplitude and the phase of a sine wave of frequency $v_{1}$ fitted to our data. The reference date for the phase $\left(t_{0}\right)$ was arbitrarily chosen on JD 2450000.00. Right: same for the September 2000 (campaign [6]) data and assuming a "period" of 2.50 days $\left(v_{1}=0.40 \mathrm{~d}^{-1}\right)$.

From their analysis of several observing campaigns with the IUE, Kaper et al. (1999) noted that the recurrence time of DACs in the UV spectrum of $\lambda$ Cep changed with time. From the October 1989 data, they found a time scale around 2.2-2.5 days, while the February 1991 and October 1991 data yielded time scales of 4.3 and 1.4 days respectively. This situation is somewhat reminiscent of the results of our Fourier analysis of the $\mathrm{He}$ II $\lambda 4686$ line of $\mathrm{BD}+60^{\circ} 2522$. Kaper et al. suggest that the wind of $\lambda \mathrm{Cep}$ is modulated by rotation and the different time scales might correspond to different integer fractions of the rotational period.
In summary, it appears that NRPs are a common feature among Oef stars and that, for lines formed in the stellar wind, profile modulation on several, sometimes variable, time scales is not unusual either.

\subsection{The role of stellar rotation}

Our analysis of $\mathrm{BD}+60^{\circ} 2522$ reveals profile variability of the double-peaked He II $\lambda 4686$ line on time scales of a few days. Both the time scale and shape of the variability pattern change 


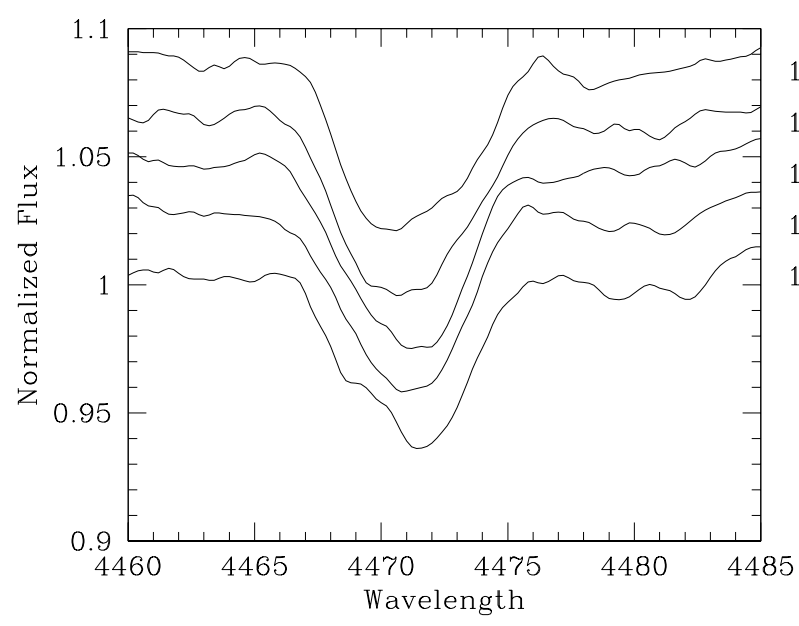

18.615

18.577

18.544

18.509

18.478

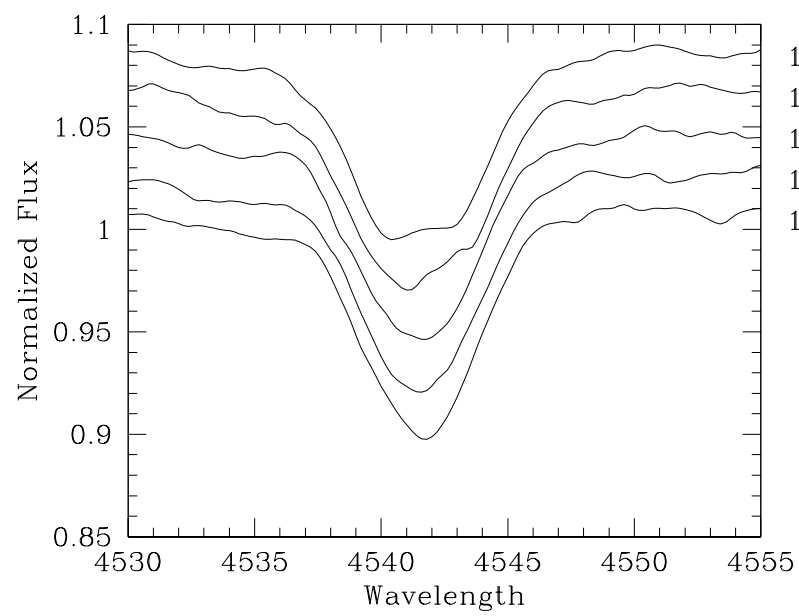

18.615 18.577 18.544 18.509 18.478

Fig. 8. Top: variations of the $\mathrm{He}$ г $\lambda 4471$ and $\mathrm{Mg}$ II $\lambda 4481$ line profiles during the night of 22-23 August 1996. The labels on the right yield the time of the observation in HJD-2450300. Consecutive spectra are shifted vertically by 0.02 . Bottom: same but for the He II $\lambda 4542$ line.

as a function of time. Therefore, it seems unlikely that the phenomenon is governed by a (single) stable clock such as the rotation of the star. Nevertheless, it is interesting to briefly consider the constraints on the rotational period of $\mathrm{BD}+60^{\circ} 2522$.

Conti \& Ebbets (1977) quoted a projected rotational velocity of $240 \mathrm{~km} \mathrm{~s}^{-1}$. More recently, Penny (1996) and Howarth et al. (1997) used IUE spectra to infer $v \sin i$ of 214 and $178 \mathrm{~km} \mathrm{~s}^{-1}$ respectively. Though these values are not exceptionally large, we note nevertheless that they place the star in the extended high velocity tail of the distribution of observed projected rotational velocities as presented by Penny (1996). This conclusion holds true regardless which of the above values we actually consider. Assuming a radius of $15 R_{\odot}$ (Howarth \& Prinja 1989), we derive an upper limit on $P_{\text {rot }}$ in the range 3.174.27 days depending on the actual value of $v \sin i$. We note that the morphology of the He II $\lambda 4686$ line in the spectrum of $\mathrm{BD}+60^{\circ} 2522$ closely ressembles the synthetic $\mathrm{H} \alpha$ profiles simulated by Petrenz \& Puls (1996) for large values of $i$. This suggests that the true rotational period could be pretty close to the upper limit derived hereabove.
A lower limit on $P_{\text {rot }}$ can be obtained from the critical rotational velocity. Adopting $\log L / L_{\odot}=5.6, T_{\text {eff }}=37500 \mathrm{~K}$ and $M=44 M_{\odot}$ (Howarth \& Prinja 1989) and using the relation from Lamers \& Leitherer (1993) to evaluate the ratio of the stellar luminosity to the Eddington luminosity, we derive a critical rotational period of 1.17 days.

The modulation of the line profile could occur with a period representing an integer fraction of the rotational period $P_{\text {rot }} / n$ if there were $n$ corotating structures in the stellar wind. The changes in the time scale of the variations might then reflect a change in the number of structures $n$ creating the modulation. However, the above constraints on $P_{\text {rot }}$ leave little room for any combination of integer numbers that might suit the various observed time scales.

These results do not imply that rotation does not contribute at all to the observed variability of the He II $\lambda 4686$ line, but they indicate that rotation cannot be the sole cause of the phenomenon at all epochs. For instance, we note that the frequency that turns up most often in our Fourier analysis $\left(v=0.34 \mathrm{~d}^{-1}\right)$ yields a "period" of 2.94 days that is rather close to the upper limit on $P_{\text {rot }}$ and this frequency might well be related to the rotational frequency.

\subsection{Towards a possible interpretation}

Let us now consider some possible scenarios that could explain the observed variability of the He II $\lambda 4686$ emission line in the spectrum of $\mathrm{BD}+60^{\circ} 2522$. First, we note that a binary scenario is rather unlikely because we find no significant $R V$ variations. Also, such a scenario would not provide an explanation for the changing periodicity. A single non-radial pulsation seems equally unlikely. In fact, the time scales observed in the variations of the He II $\lambda 4686$ line are much too long compared to the time scales of the variations of the absorption lines.

It seems therefore most likely that the phenomenon is associated with some transient large scale structure in the stellar wind. The lack of a single time scale in the modulation of the He II $\lambda 4686$ line suggests that the phenomenon might not be related to corotating structures. Such corotating structures are predicted for instance by the so-called corotating interaction region model (Cranmer \& Owocki 1996; Owocki 1999) and are believed to generate the cyclical modulation of the P-Cygni absorptions in UV resonance lines observed for a number of $\mathrm{OB}$ stars (e.g. Fullerton et al. 1997).

Let us emphasize that the double-peaked morphology of the He II $\lambda 4686$ line clearly suggests that the stellar wind must be rotating at an appreciable velocity out to at least the radius of the formation region of this line. For instance, the wind could be forced to corotate with the star through the effect of a moderate stellar magnetic field. This situation could lead to some confinement of the stellar wind in latitude (see e.g. Babel \& Montmerle 1997) and there is some observational evidence for an equatorial compression of the stellar wind of Oef stars. In fact, such a compression of the wind of $\zeta$ Pup was put forward by Harries \& Howarth (1996) based on their spectropolarimetric observations of the $\mathrm{H} \alpha$ line in the spectrum of this star. 
A variation of the global mass loss rate would change the extent of the corotating confined wind and could therefore induce some changes in the time scale of the variability. However, in case of a change of the overall mass loss rate, we would also expect to see epoch dependent changes in the emission strengths. The mean profiles displayed in Fig. 5 and the lack of a clear long term trend in the EWs listed in Table 3 do not support this idea.

It is worth pointing out that the pattern of the emission line profile variability in September 2000 is very much reminiscent of the model calculations of recurrent line profile variations presented by Lépine (2002). In these simulations, Lépine assumes a large number of discrete locally optically thick wind inhomogeneities moving around a common rotation axis. This model is designed to simulate the line profile variations arising from a perturbation at the surface of a rotating Wolf-Rayet star core that triggers waves that propagate outwards in the wind and create several overdense rotating "spiral arms". The main difference between the Lépine model and our observations of the $\mathrm{He}$ II $\lambda 4686$ line in the spectrum of $\mathrm{BD}+60^{\circ} 2522$ is the lack of a single stable period in the observed modulation.

A possible explanation of the modulation of the $\mathrm{He}$ II $\lambda 4686$ line in the spectrum of $\mathrm{BD}+60^{\circ} 2522$ might be a density perturbation propagating across (rather than just rotating with) the confined part of the wind. We could imagine this as some sort of analogue of the large scale oscillations of Be-like disks. This density perturbation could be triggered by an episodic enhancement of the local mass loss at the tip of a large amplitude NRP wave. A slightly similar mechanism, "NRP wave breaking", was proposed by Osaki (1999) to explain the episodic mass loss in Be stars. Such a large amplitude NRP wave could arise e.g. from beating among various NRP modes. While this scenario could easily account for the lack of a single stable period (through the effect of the propagation velocity of the perturbation and the interplay of various clocks: pulsations, rotation...), it seems more difficult to explain the changing pattern of the TVS. For instance, if a density wave moves around the star, why would it not affect the absorption and the emission components in a similar manner? One possibility could be that the density perturbation affects the absorption column only as long as it remains close to the stellar surface whilst the impact on the emission lines would be larger when the perturbation has moved outwards, but this is admittedly still rather speculative.

\section{Summary and concluding remarks}

Our time series of spectra of the O6.5ef star BD $+60^{\circ} 2522$ show that the double-peaked He II $\lambda 4686$ emission line undergoes strong profile variability on time scales of 2-3 days similar to the variations seen in two other Oef stars, $\zeta$ Pup and $\lambda$ Cep. Both the time scale as well as the pattern of the variations in $\mathrm{BD}+60^{\circ} 2522$ turn out to be epoch dependent, suggesting that the phenomenon is not related to the sole effect of stellar rotation. On the other hand, the absorption lines display line profile variability on time scales of a few hours which are probably indicative of non-radial pulsations. Dedicated observations with a better time resolution are needed to confirm the latter finding and to quantify the time scales of these pulsations.
It seems very likely that the wind of $\mathrm{BD}+60^{\circ} 2522$ is not spherically symmetric. Out to the formation region of the He II $\lambda 4686$ line, the wind appears rather as some sort of corotating equatorially confined envelope. In this context, it would be extremely interesting to investigate the possible presence of a stellar magnetic field in $\mathrm{BD}+60^{\circ} 2522$. Evidence for a stellar magnetic field might be obtained through the detection of a synchrotron (i.e. non-thermal) radio emission. A detection of a non-thermal radio emission from any of the Oef stars might therefore be of primary importance for our understanding of the Oef phenomenon. We note however, that at least for $\zeta$ Pup, the VLA observations by Bieging et al. (1989) revealed a purely thermal radio spectrum.

Acknowledgements. We wish to express our thanks to Dr. Jean Manfroid for taking some of the spectra of the September 2000 campaign and to the referee Dr. P.S. Conti for the careful reading of the manuscript. We are greatly indebted to the Fonds National de la Recherche Scientifique (Belgium) for multiple assistance including the financial support for the rent of the OHP telescope in 1999, 2001 and 2002 through contract 1.5.051.00 "Crédit aux Chercheurs" FNRS. The travels to OHP for the observing runs were supported by the Ministère de l'Enseignement Supérieur et de la Recherche de la Communauté Française. This research is also supported in part by contracts P4/05 and P5/36 "Pôle d'Attraction Interuniversitaire" (SSTCBelgium) and through the PRODEX XMM-OM Project. We would like to thank the staff of the Observatoire de Haute Provence for their technical support during the various observing runs. The SIMBAD database has been consulted for the bibliography.

\section{References}

Baade, D. 1988, in O Stars and Wolf-Rayet Stars, ed. P. S. Conti, \& A.B. Underhill, NASA SP-497, 137

Babel, J., \& Montmerle, T. 1997, ApJ, 485, L29

Berghöfer, T. W., Baade, D., Schmitt, J. H. M. M., et al. 1996, A\&A, 306, 899

Bieging, J. H., Abbott, D. C., \& Churchwell, E. B. 1989, ApJ, 340, 518

Chavarría-K., C., Jäger, C., \& Leitherer, C. 1987, in Circumstellar Matter, ed. I. Appenzeller, \& C. Jordan (Reidel, Dordrecht), Proc. IAU Symp., 122, 445

Christopoulou, P. E., Goudis, C. D., Meaburn, J., Dyson, J. E., \& Clayton, C. A. 1995, A\&A, 295, 509

Conti, P. S. 1973, ApJ, 179, 181

Conti, P. S., \& Alschuler, W. R. 1971, ApJ, 170, 325

Conti, P. S., \& Leep, E. M. 1974, ApJ, 193, 113

Conti, P. S., \& Ebbets, D. 1977, ApJ, 213, 438

Cranmer, S. R., \& Owocki, S. P. 1996, ApJ, 462, 469

de Jong, J. A., Henrichs, H. F., Schrijvers, C., et al. 1999, A\&A, 345, 172

Doroshenko, V. T. 1972, SvA, 16, 402

Eversberg, T., Lépine, S., \& Moffat, A. F. J. 1998, ApJ, 494, 799

Fullerton, A. W. 1999, in Variable and Non-spherical Stellar Winds in Luminous Hot Stars, ed. B. Wolf, O. Stahl, \& A. W. Fullerton, Lecture Notes in Physics, 523, 3

Fullerton, A. W., Gies, D. R., \& Bolton, C. T. 1996, ApJS, 103, 475

Fullerton, A. W., Massa, D. L., Prinja, R. K., Owocki, S. P., \& Cranmer, S. R. 1997, A\&A, 327, 699 
Gillet, D., Burnage, R., Kohler, D., et al. 1994, A\&AS, 108, 181

Harries, T. J., \& Howarth, I. D. 1996, A\&A, 310, 533

Henrichs, H. F. 1991, in Rapid variability of OB stars: Nature and diagnostic value, ed. D. Baade, Proc. ESO Workshop 36, 199

Howarth, I. D., \& Prinja, R. K. 1989, ApJS, 69, 527

Howarth, I. D., Prinja, R. K., \& Massa, D. 1995, ApJ, 452, L65

Howarth, I. D., Siebert, K. W., Hussain, G. A. J., \& Prinja, R. K. 1997, MNRAS, 284, 265

Kaper, L., Henrichs, H. F., Fullerton, A. W., et al. 1997, A\&A, 327, 281

Kaper, L., Henrichs, H. F., Nichols, J. S., \& Telting, J. H. 1999, A\&A, 344,231

Kuan, P., \& Kuhi, L. V. 1976, PASP, 88, 128

Lamers, H. J. G. L. M., \& Leitherer, C. 1993, ApJ, 412, 771

Lépine, S. 2002, in Interacting Winds from Massive Stars, ed. A. F. J. Moffat, \& N. St-Louis, ASP Conf. Ser., 260, 185

Moffat, A. F. J., \& Michaud, G. 1981, ApJ, 251, 133
Osaki, Y. 1999, in Variable and Non-spherical Stellar Winds in Luminous Hot Stars, ed. B. Wolf, O. Stahl, \& A. W. Fullerton, Lecture Notes in Physics, 523, 329

Owocki, S. P. 1999, in Variable and Non-spherical Stellar Winds in Luminous Hot Stars, ed. B. Wolf, O. Stahl, \& A. W. Fullerton, Lecture Notes in Physics, 523, 294

Penny, L. R. 1996, ApJ, 463, 737

Petrenz, P., \& Puls, J. 1996, A\&A, 312, 195

Rauw, G., Morrison, N. D., Vreux, J.-M., Gosset, E., \& Mulliss, C. L. 2001, A\&A, 366, 585

Reid, A. H. N., \& Howarth, I. D. 1996, A\&A, 311, 616

Underhill, A. B., Gilroy, K. K., \& Hill, G. M. 1989, AJ, 98, 1063

Walborn, N. R. 1973, AJ, 78, 1067

Werner, K., \& Rauch, T. 2001, in Eta Carinae and Other Mysterious Stars: The hidden opportunities of emission spectroscopy, ed. T. R. Gull, S. Johansson, \& K. Davidson, ASP Conf. Ser., 242, 229 